\title{
Nongenomic Mechanisms of PTEN Regulation
}

\author{
Jimmie E. Fata, ${ }^{1,2,3}$ Shawon Debnath, ${ }^{1,3}$ Edmund C. Jenkins Jr., ${ }^{1,4}$ and Marcia V. Fournier ${ }^{5}$ \\ ${ }^{1}$ Department of Biology, College of Staten Island, 2800 Victory Boulevard, Staten Island, NY 10314, USA \\ ${ }^{2}$ Biology Doctoral Program, City University of New York Graduate Center, 365 Fifth Avenue, New York, NY 10016, USA \\ ${ }^{3}$ Biochemistry Doctoral Program, City University of New York Graduate Center, 365 Fifth Avenue, New York, NY 10016, USA \\ ${ }^{4}$ Biology Doctoral Program, City University of New York Graduate Center, 365 Fifth Avenue, New York, NY 10016 USA \\ ${ }^{5}$ BIOARRAY Therapeutics Inc., Venture Development Center, UMASS, 100 Morrissey Boulevard, Boston, MA 02125, USA
}

Correspondence should be addressed to Jimmie E. Fata, jimmie.fata@csi.cuny.edu

and Marcia V. Fournier, marcia.fournier@yahoo.ca

Received 2 November 2011; Revised 16 January 2012; Accepted 17 January 2012

Academic Editor: Andre Van Wijnen

Copyright (c) 2012 Jimmie E. Fata et al. This is an open access article distributed under the Creative Commons Attribution License, which permits unrestricted use, distribution, and reproduction in any medium, provided the original work is properly cited.

\begin{abstract}
A large amount of data supports the view that PTEN is a bona fide tumor suppressor gene. However, recent evidence suggests that derailment of cellular localization and expression levels of functional nonmutated PTEN is a determining force in inducing abnormal cellular and tissue outcomes. As the cellular mechanisms that regulate normal PTEN enzymatic activity resolve, it is evident that deregulation of these mechanisms can alter cellular processes and tissue architecture and ultimately lead to oncogenic transformation. Here we discuss PTEN ubiquitination, PTEN complex formation with components of the adherens junction, PTEN nuclear localization, and microRNA regulation of PTEN as essential regulatory mechanisms that determine PTEN function independent of gene mutations and epigenetic events.
\end{abstract}

\section{PTEN: A Unique Dual-Specificity Phosphatase}

PTEN (phosphatase and tensin homolog deleted on chromosome ten)/MMAC (mutated in multiple advanced cancers) has been identified simultaneously by two research groups as a candidate tumor suppressor gene located at $10 \mathrm{q} 23$ and encoding 403 amino acids $[1,2]$. Another group identified the same gene in the search for new dual-specific phosphatases and named it TEP-1 (TGF- $\beta$ regulated and epithelial cell-enriched phosphatase) [3]. PTEN is one of the most common targets of mutation in human cancer, with a mutation frequency approaching that of the tumor suppressor gene p53, and it is also mutated in inherited cancer predisposition disorders. PTEN belongs to the protein tyrosine phosphatase family with phosphatase activity on both lipids and proteins. PTEN's lipid phosphatase catalyzes the conversion of phosphatidylinositol-(3,4,5)-triphosphate $\left(\mathrm{PIP}_{3}\right)$ to phosphatidylinositol-4,5-bisphosphate $\left(\mathrm{PIP}_{2}\right)$ [4, 5] and plays an important role in the PI3K pathway by catalyzing degradation of $\mathrm{PIP}_{3}$ generated by PI3K. This inhibits PI3K downstream targets, mainly PKB-Akt [6-10]. It should be noted, however, that lipid phosphatase attenuated or inactive PTEN mutants have been reported to still retain some tumor suppressing properties [11-15]. So far there is no report of redundancy for PTEN function, which could explain the high frequency with which PTEN inactivation is selected during tumor development [16]. By virtue of PTEN's ability to attenuate and control the extent of PI3K signaling, PTEN influences many cellular functions, including cell growth, survival, proliferation, and metabolism [8]. PTEN contributes to cell cycle regulation by blocking cells entering the S-phase of the cell cycle and by upregulation of p27kip1, which is recruited into the cyclin E/cdk2 complex [17], and downregulation of cyclin D1 [18]. Exogenous PTEN can suppress the growth of cells with mutated PTEN alleles [19], but the data of Tamura et al. [20] also indicates that this tumor suppressor has biological cell activity unrelated to growth. In contrast to many other tumor 
suppressors, which appear to have only nuclear roles, PTEN also appears to function in regulating dynamic cell surface interactions that involve integrins, FAK, cell migration, and the cytoskeleton [21-23].

In tumor tissue, proper function of PTEN acts as a tumor suppressor primarily through the ability to suppress proliferation and decrease cell survival. The frequent loss of PTEN function, through deletion, mutations, and/or decreased expression, is observed in hereditary cancers as well as sporadic cancers [8]. In many sporadic cancers, including breast cancer, PTEN is commonly found mutated at one allele. These monoallelic mutations of PTEN have been suggested to be as prevalent as p53 mutations found in most cancers and support the belief that PTEN is a bona fide tumor suppressor capable of controlling tumor initiation and progression. Germline mutations of PTEN are evident in hereditary autosomal dominant cancer syndromes, which have been collectively termed PTEN hamartoma tumor syndromes (PHTSs) [24, 25]. Many of these syndromes show increased risk of cancer occurring in the breast, thyroid, and endometrial tissues. It becomes apparent that some tissues are more prone to tumor initiation and progression in the absence of one PTEN allele, while other tissues often require both alleles deleted.

In the absence of germline and monoallelic mutations, PTEN protein levels have been found to be progressively lost during cancer progression [26]. A number of mechanisms, other than gene mutation and deletion, contribute to the loss or the decrease of PTEN protein levels in human cancers [27-31]. Proposed mechanisms for progressive loss of PTEN expression, in the absence of mutations, have been attributed to epigenetic events such as promoter methylation. Moreover, a large number of studies have indicated that posttranslational modifications on PTEN effects the protein's function, that is, phosphorylation and ubiquitination decrease PTEN protein levels, while oxidation and acetylation reduce PTEN activity [32]. Other reports suggest that E-cadherin expression or function may be an initiating cause of loss of PTEN expression in cancers, such as those that frequently occur in breast cancer, where PTEN expression is lost without identifiable mutations in the PTEN gene itself. Recently, several reports have indicated that PTEN shuttles between the nuclear and cytoplasmic compartments and that PTEN nuclear pool may have function to maintain genome integrity and cell-cycle control. Furthermore, the emerging field of microRNAs has revealed that PTEN is a direct and indirect target of some of these noncoding RNAs. Here we discuss the regulation of PTEN function by mechanisms other than gene mutations and epigenetic events as they relate to cellular function and oncogenic transformation (Figure 1).

\section{Regulation of PTEN Protein Levels}

The relevance of downregulated PTEN protein levels, as opposed to a complete loss of PTEN, is best observed in mouse models where PTEN is genetically manipulated [3337]. In mice heterozygous for PTEN, where PTEN levels are essentially "50\%" less when compared to wild type mice, a prevalence of tumors in the endometrium, thyroid, prostate, and liver as well as lymphomas was found. In contrast, mice with homozygous deletion of PTEN exhibited embryonic lethality by day 9.5 and displayed defective chorioallantoic development. Heterozygous deletions of PTEN indicate that PTEN haploinsufficiency predisposes some epithelial cells to becoming cancerous and this may explain why a large percentage of human epithelial tumors do not show any signs of gene mutation or deletion and instead retain wild-type copies of PTEN. PTEN transgenic mice, that express a range of PTEN levels (hypomorphs) all lower than wild type levels, have provided strong evidence that tissue response to PTEN function is truly dose-dependent $[35,37]$.

In some cancers, like nonsmall cell lung carcinoma (NSCLC), where PTEN expression is reduced or lost in 55\%$74 \%$ of patients, genetic alteration such as loss of heterozygosity and epigenetic silencing were not good predictors of PTEN protein levels $[29,30]$. With NSCLC, PTEN down regulation via ubiquitin-mediated degradation appears to be the primary mechanism of loss of PTEN function. The E3-ubiquitin ligase NEDD4 (neural precursor cell-expressed, developmentally downregulated 4-1) is overexpressed in $80 \%$ of NSCLC compared to normal tissue and has been shown to promote PTEN protein ubiquitin-mediated degradation and control PTEN stability in mouse tissue [28]. In light of these findings, NEDD4 overexpression in NSCLC has been suggested to be the primary factor in driving PTEN levels down in a large proportion of these cancers. In breast cancer, where PTEN protein is reduced in as much as $50 \%$ of cases, genetic and epigenetic alterations of the PTEN locus are rare, again suggesting that PTEN downregulation is occurring via a posttranslational event [38-40]. Similar to NSCLC, NEDD4 has been implicated in regulating PTEN turnover in breast cancer, a process that is inhibited by RAK phosphorylation of PTEN at Tyr-336 [41]. Unlike the Rak phosphorylation site on PTEN (Tyr-336), which stabilizes PTEN protein, phosphorylation at Thr-366, Ser-370, Ser380, Thr-382, and Ser-385 has been attributed to attenuating PTEN stability [42]. It should be noted that Rak, which exhibits LOH in approximately $30 \%$ of breast cancers [43], has been subsequently termed a bona-fide tumor suppressor gene in breast cancer [41].

NEDD4 ubiquitin-ligase activity, which downregulates PTEN protein levels, is enhanced by the small endosomal PY-motif containing membrane proteins Ndfip1 and Ndfip2 [44]. This suggests that in normal cells the PTEN axis is balanced by opposing actions of Rak-induced PTEN stability and Ndfip1/Ndfip2 enhanced NEDD4-directed PTEN degradation-a shift in favor of NEDD4 activity predisposes cells to oncogenic transformation. Although strong evidence points to NEDD4 as an important regulator of PTEN protein levels in a number of cancer cells (bladder, gastric, and colorectal) $[45,46]$, gene knockout studies have challenged this assumption since in mice lacking NEDD4, PTEN protein levels remain stable with no evidence of increased stability in multiple cell types tested [47, 48]. Moreover, evidence from both in vitro and in vivo studies indicates that PTEN ubiquitination is still evident even in the absence of NEDD4. 


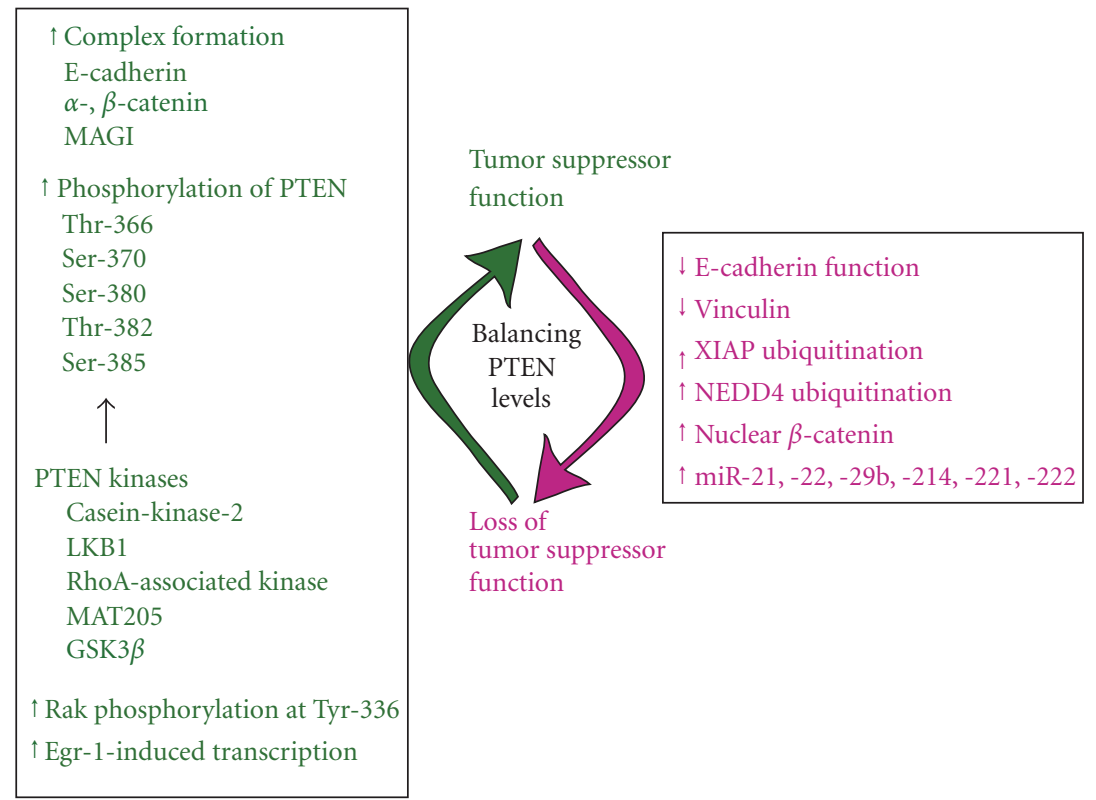

FIGURE 1: Mechanisms other than gene mutations and epigenetic silencing that regulate PTEN levels and ultimately its tumor suppressor function.

Other E3 ligases such as the X-linked inhibitor of apoptosis protein (XIAP) may also contribute in regulating PTEN protein levels. XIAP knockdown studies lower PTEN ubiquitination and upregulate PTEN stability, and conversely, overexpression of XIAP leads to PTEN poly-ubiquitination and lowered PTEN protein levels [49]. XIAP is a member of the inhibitor of apoptosis (IAP) family of proteins, and aside from its ability to act as E3-ligase, it is also capable of suppressing caspase-dependent cell death and is often upregulated in cancers $[50,51]$. The phosphorylation state of PTEN also contributes to the regulation of PTEN subcellular localization and protein levels. PTEN phosphorylation at serine 380 (Ser-380) and threonines 382/383 (Thr-382/383) within its C-terminal tail strongly influences PTEN protein stability and its localization to the cell membrane. Several kinases, including casein kinase-2, LKB1, RhoA-associated kinase, the microtubule associated kinase MAT205, and GSK3 $\beta$, have been reported to phosphorylate PTEN [52]. In light of the multiple pathways that may regulate PTEN protein levels, it becomes increasingly evident that these mechanisms can directly affect tumor initiation and progression.

\section{PTEN and E-cad/MAGI}

Calcium-dependent homophilic binding of the adhesion protein E-cadherin is vital for the cell-cell interaction found in epithelial tissue, while loss of E-cadherin expression is associated with transformation and metastatic cancers [5355]. It has been demonstrated that PTEN interacts with cell adhesion molecules such as E-cadherin and MAGI-2 protein to inhibit migration and proliferation [56-59]. Although generally considered a cell-adhesion molecule, E-cadherin is also part of an active signaling network affecting a variety of cellular processes such as invasion and proliferation [6065]. For instance, suppression of E-cadherin expression has been linked to the downregulation of PTEN expression and subsequent activity [66], while we have shown that restoration of E-cadherin increased PTEN protein levels in E-cadherin null breast cancer cells [67]. A cross-talk exists between PTEN and E-cadherin since PTEN also stabilizes Ecadherin at the adherens junction [68].

MAGI (membrane-associated guanylate-kinase inverted) family members are multidomain scaffolding proteins with multiple sites for protein interaction. There are multiple splice variants of MAGI; MAGI-1a, MAGI-1b, MAGI-2, and MAGI-3, where each is expressed in a tissue-specific manner. MAGI proteins are part of a PDZ subfamily of proteins called MAGUK (membrane-associated guanylate kinases). Members of this family, including MAGI-2 and -3, were shown to directly bind PTEN in yeast two-hybrid screens $[56,69,70]$. Soon after, mutational analysis showed that phosphorylation at Thr-382/383 greatly increases affinity of PTEN for MAGI-2 [58]. Kotelevets et al. put forth the idea of a signalosome composed of the scaffolding protein MAGI, $\alpha$ - and $\beta$-catenin, PTEN, PI3K, and E-cadherin [71]. In this complex PTEN is believed to be stabilized and protected from degradation, thus increasing its presence in the cytosol without affecting mRNA levels $[57,59]$. Indeed, the importance of the association of the E-cadherin via the signalosome to the cytoskeleton is underscored by the loss of vinculin, which reduced E-cadherin mediated cell-cell binding $[57,72]$ and subsequently decreased PTEN protein levels [57]. PTEN levels were restored in vin $^{-1-}$ cells by the overexpression of MAGI-2 or by expressing an E-cadherin- $\alpha$-catenin fusion protein. [57]. Our previous work has shown that PTEN is recruited to cell-cell interactions with E-cadherin, MAGI2 , and $\beta$-catenin in a nonmalignant mammary acini model 
in laminin-rich extracellular matrix. In this model, either inhibition of E-cadherin function or reduction of PTEN protein levels abrogated acini organization and proliferation control suggesting that PTEN roles in cell adhesion and proliferation may be interconnected [67]. The surprising sensitivity of mammary cells to the PTEN regulatory node may explain why it is so low in a variety of solid tumors where tissue structure is compromised. These findings suggest that PTEN-dependent changes in PI3K signaling may operate in conjunction with other E-cadherin-dependent processes to cause cessation of cell growth.

$\beta$-catenin is part of the cadherin-catenin complex and indirectly connects E-cadherin to the cytoskeleton. Studies have suggested that $\beta$-catenin negatively regulates PTEN transcription by blocking early growth response gene 1 (Egr1) [66]. Egr1 has been shown to activate PTEN transcription by directly binding to the Egrl binding motif in the PTEN promoter [73]. Taken together, a picture arises depicting the relationship between E-cadherin, PTEN, MAGI, and loss of cell-cell adhesion in cancer. When present, E-cadherin stabilizes the adherens junction complex, including the signalosome, which sequesters $\beta$-catenin at the plasma membrane. Thus, $\beta$-catenin cannot translocate to the nucleus and inhibit Egr1 transcription, the effect of which is continued PTEN transcription. Additionally, PTEN is protected in the signalosome from proteosomal degradation, resulting in sustained PTEN protein levels in the cytosol and subsequently negative regulation of the PI3K/AKT pathway. Loss of E-cadherin expression in cancer causes disruption of the signalosome, thus freeing $\beta$-catenin and PTEN from the MAGI scaffolding protein. The net effect of this is the proteosomal degradation of PTEN and the inhibition of PTEN transcription by $\beta$-catenin that has translocated to the nucleus.

\section{Nuclear PTEN}

The existence of nuclear PTEN was reported in a number of cell models including primary neurons and endothelial cells [74], myoepithelial cells of normal breast ducts [75], and normal follicular thyroid cells [76]. In general stronger PTEN nuclear staining has been observed in normal cells when compared to tumor cells [52] and is thought to directly correlate with cell differentiation [77]. The presence of nuclear PTEN was at first thought to be an artifact based on immunohistochemical techniques; however, a proposed role for nuclear PTEN was provided from studies examining melanoma tumors where a decrease in PTEN nuclear levels correlated with an increase in the proliferation status of tumor cells [78]. As well, an investigation with colorectal adenocarcinoma showed a gradual decrease in nuclear PTEN expression from normal to subsequent progression stages of metastasis [79]. Subcellular fractionation and immunocytochemistry studies have confirmed that nuclear translocation of PTEN in the human breast carcinoma cell line MCF7 was cell cycle dependant [80]. This cell line has also demonstrated that a rise in nuclear PTEN level occurs at G0-G1 with a decrease in nuclear PTEN level in S-phase, indicating nuclear PTEN to be involved as a cell cycle modulator [81]. Further studies using the MCF7 cell line found that nuclear PTEN is required for cell cycle arrest and cytoplasmic PTEN is required for apoptosis [81]. In contrast to breast cancer cells, increased level of nuclear PTEN was associated with G2 arrest in melanoma cells instead of G1 [82]. These findings indicate that nuclear localization of PTEN is associated with decreased proliferation. These studies and others indicated that increased nuclear translocation of PTEN is associated with PTEN tumor-suppressing activity and nuclear PTEN depletion correlates with increased tumor progression [76, 78, 83, 84] (Figure 2).

The absence of a "true" nuclear localization signal within PTEN has led to a number of alternative mechanisms for nuclear entry of PTEN [85]. One mechanism may be simple diffusion, which was found with a GFP-PTEN fusion protein construct entering the nucleus of HeLa cells [86]. It should be noted that proteins with molecular mass greater than $60 \mathrm{kDa}$ are generally prohibited from nuclear entry by diffusion $[87,88]$. However, diffusion does not explain the differential distribution pattern of PTEN in differentiated/nondividing resting cells and tumor cells. Therefore, it has been suggested that an active transport system for PTEN nuclear trafficking must exist. One such mechanism may involve the small GTPase RAN, which is a wellstudied regulator of importin-mediated nuclear transport [89]. Upon investigating this mechanism, it was found that a GTPase deficient dominant negative RANQ69L mutant excluded nuclear entry of PTEN [90]. It should be noted that this RAN-dependent mechanism of PTEN nuclear import is also associated with increased cellular apoptosis in the human glioblastoma cell line U87MG. Another mechanism of active nuclear import of PTEN may involve the Major Vault Protein (MVP), which is a carrier molecule involved in nuclear-cytoplasmic transport of molecules [91]. Evidence was provided that PTEN binding to MVP promoted nuclear translocation of PTEN in yeast-two hybrid system [92]. Later it was confirmed in 293T and HeLa cell lines that it was a $\mathrm{Ca}^{+2}$-regulated interaction and the binding was specific between the C2 PTEN domain and the calcium binding motif (EF-hand pair) of MVP [85]. Other mechanisms of PTEN nuclear import may involve posttranslational modifications of PTEN. For instance, polyubiquitination of PTEN results in PTEN degradation, whereas monoubiquitinated PTEN at K289 undergoes nuclear translocation [45]. Phosphorylation of PTEN is also another posttranslational modification of PTEN that has been shown to directly affect nuclear translocation. The C-terminal domain of PTEN possesses important phosphorylation sites (Ser-380, Thr-382/383, Ser385) which are involved in regulation of PTEN stability, activity, and localization [42, 93-98]. Phosphorylation of PTEN at Ser-380 is upregulated during oxidative stress and associates with an accumulation of nuclear PTEN [98]. The outcome of this accumulation of nuclear PTEN leads to growth arrest, cellular apoptosis, and a reduction of reactive oxygen species production [98]. Findings have also indicated that nuclear localization of PTEN is regulated by the PI3K pathway. Specifically, cells with dominant negative Akt mutants or those treated with inhibitors of PI3K mTOR suppress PTEN nuclear transport, while siRNA silencing 
High nuclear/cytoplasmic ratio

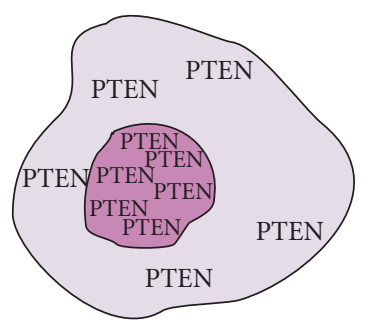

High in normal cells Associates with cell differentiation Reduces ROS

Decreases cell cycle progression Associates with cell cycle arrest Increases APC-CDH1 complex

Increased tumor suppressive activity
Low nuclear/cytoplasmic ratio

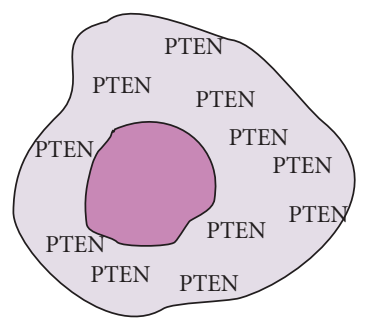

High in cancer cells

Associates with increased proliferation

Associates with cell cycle arrest

Decreases APC-CDH1 complex

Decreased tumor suppressive activity

FIGURE 2: Cell-characteristics associated with the presence or absence of nuclear PTEN.

for S6K 1/2 (protein kinase) also blocks PTEN nuclear entry. Together these data suggest that PI3K/Akt/mTOR/S6K activation leads to PTEN nuclear transport [99]. Finally, it has been reported that LKB1/CaMKK- $\left(\mathrm{Ca}^{+2} /\right.$ calmodulindependant protein kinase) mediated activation of AMPK $\alpha 1 / 2$ can bypass the inhibition of PTEN nuclear entry achieved with mTOR/S6K downregulation [100] and thus provides another mechanism for PTEN entering the nucleus.

In seminal work, the functional significance of nuclear PTEN with regard to proliferation and cell cycle control has been revealed [101]. In this particular study, it was found that PTEN nuclear exclusion rather than its phosphatase inactivation was responsible for decreased activation of the APC(Anaphase-promoting complex/cyclosome-) CDH1 tumor suppressor complex. Essentially, nuclear PTEN increases the activity of APC/C complex and induces its association with $\mathrm{CDH} 1$. APC/C imparts a major regulatory control on the cell cycle from mitosis to late G1 phase $[102,103]$, and its functional activity is maintained through its interaction with CDC20 and CDH1, while CDH1 activity is restricted between late mitosis and G1 [104, 105]. The APC-CDH1 complex is essential for cell-cycle regulatory control and tumor-suppressive activity [101]. By virtue of regulating the APC/CDH1 complex nuclear PTEN becomes an important factor in determining cellular outcome and proliferative status. This is an important finding related to cancer therapy since, it has been also shown that loss of PTEN sensitizes cells to pharmacological inhibition of PLK1 and Aurora kinases.

\section{Regulation of PTEN by MicroRNAs}

MicroRNAs (miRNAs) are short 18-25 nucleotide long noncoding RNAs involved in posttranscriptional regulation of gene expression [106]. They negatively regulate target gene expression through complimentary binding to the $3^{\prime}$ untranslated region (UTR) of mRNAs [107-111]. MicroRNAs have gained importance in development and progression of several types of cancer that can be explained by their ability to modulate the expression of gene products involved in cell migration, invasion, and apoptosis [112, 113]. Overexpression of certain microRNAs like miR-21, miR-10b, miR-373, and miR-155 has shown to promote metastasis in cancer whereas downregulation of other miRNAs such as miR-31, let-7, miR-146, and miR-193b has been shown to promote tumor growth, metastasis, and invasion [114-117].

In 2007, Meng et al. reported overexpression of miR-21 in human hepatocellular cancer using miRNA microarray assay [27]. Inhibition of miR-21 was accompanied by an increase in PTEN expression with a decrease in cell migration, invasion and cell proliferation. Downmodulation of miR-21 also affected the downstream effectors of PTEN such as FAK phosphorylation as well as MMP-2/9 expression involved in cell migration and invasion. This original work supported that PTEN was a direct target of miR-21. Later miR-21 was also reported to be overexpressed in nonsmall cell lung cancer cells (NSCLCs) compared to adjacent nontumor cells with an inverse corelation to PTEN expression [118]. NSCLC cell lines transfected with miR-21 inhibitor resulted in increased luciferase reporter activity for PTEN 3' UTR, suggesting that miR-21 binds to the $3^{\prime}$ UTR of PTEN mRNA. Additionally, an elevation in PTEN protein level was detected without any effect on PTEN mRNA levels. This work supported the hypothesis that miR-21 down regulates posttranscriptional expression of PTEN. Further support for the role of miR-21 was provided by an in vivo mouse model in which miR-21 was knocked out. This increased the expression of several miR-21 target genes including PTEN [119]. In contrast to these studies, upregulation of miR-21 in normal and tumor breast cells did not associate with a detectable change in PTEN levels as determined by miRNA in situ hybridization techniques [120]. Recent work has demonstrated that the oncogenicity of miR-21 oncomir (oncogenic miRNA) could be cell and tissue dependant and must be contextualized to a particular disease prior to consideration as a therapeutic target [121].

MicroRNAs other than miR-21 have been shown to be involved in various biological events and disease through 
PTEN regulation. For instance, MiR-214 is involved in cell survival [122] and cell apoptosis [123] and has been found to be overexpressed in gastric cancer cells compared to normal gastric mucosal cell lines using real-time PCR techniques [124]. Transient transfection with antisense miRNA-214 oligonucleotides downregulated miR-214 expression with a significant increase in PTEN expression. Flow cytometry revealed G1 increases and S-phase decreases in gastric cancer cells when miRNA-214 was downregulated. PTEN was also reported to be the direct target of miR-29b in human breast cancer cells [125]. The breast cancer cell line MDA-MB231, which migrates and invades faster than MCF7 cells, was found to display higher miR-29b levels compared to MCF7 cells. Inhibition of miR-29b in MDA-MB-231 cells increased PTEN expression, which promoted apoptosis and reduced cell migration and invasion. It was also reported that knockdown of miR-221 and miR-222 in multiple cancer cell lines upregulated PTEN expression suppressed of AKT activity with enhanced radio sensitivity in tumor cells [126]. The previous findings have indicated that PTEN is a target of a number of microRNAs and that cancer cells upregulate these regulatory RNAs to downregulate the tumor suppressor characteristics of PTEN.

\section{Conclusions}

There is ample evidence that the full functionality of PTEN is modulated by alternative mechanisms beyond gene mutations and epigenetic processes. For instance a number of tissue-specific cancers strongly associate with PTEN deregulation at the gene expression level, changes in PTEN posttranslational modifications, miss-guided PTEN subcellular localization, and PTEN-specific microRNA upregulation. Determining whether these alternative mechanisms are causal or a consequence of tumor initiation and progression or whether they produce tissue-specific effects is still an ongoing area of important research. Clinically, as PTEN-regulating pathways become fully resolved, essential factors will arise, hopefully providing new targets for the development of novel and effective anticancer therapies and diagnostic tools.

\section{References}

[1] J. Li, C. Yen, D. Liaw et al., "PTEN, a putative protein tyrosine phosphatase gene mutated in human brain, breast, and prostate cancer," Science, vol. 275, no. 5308, pp. 1943-1947, 1997.

[2] P. A. Steck, M. A. Pershouse, S. A. Jasser et al., "Identification of a candidate tumour suppressor gene, MMAC1, at chromosome 10q23.3 that is mutated in multiple advanced cancers," Nature Genetics, vol. 15, no. 4, pp. 356-362, 1997.

[3] D. M. Li and H. Sun, "TEP1, encoded by a candidate tumor suppressor locus, is a novel protein tyrosine phosphatase regulated by transforming growth factor $\beta$," Cancer Research, vol. 57, no. 11, pp. 2124-2129, 1997.

[4] S. J. Leevers, B. Vanhaesebroeck, and M. D. Waterfield, "Signalling through phosphoinositide 3-kinases: the lipids take centre stage," Current Opinion in Cell Biology, vol. 11, no. 2, pp. 219-225, 1999.
[5] T. Maehama and J. E. Dixon, "The tumor suppressor, PTEN/ MMAC1, dephosphorylates the lipid second messen-ger, phosphatidylinositol 3,4,5-trisphosphate," Journal of Biological Chemistry, vol. 273, no. 22, pp. 13375-13378, 1998.

[6] P. L. M. Dahia, "PTEN, a unique tumor suppressor gene," Endocrine-Related Cancer, vol. 7, no. 2, pp. 115-129, 2000.

[7] A. Hlobilková, J. Knillová, J. Bártek, J. Lukás, and Z. Kolár, "The mechanism of action of the tumour suppressor gene PTEN," Biomedical papers of the Medical Faculty of the University Palacký, Olomouc, Czechoslovakia, vol. 147, no. 1, pp. 19-25, 2003.

[8] S. Zhang and D. Yu, "PI(3)king apart PTEN's role in cancer," Clinical Cancer Research, vol. 16, no. 17, pp. 4325-4330, 2010.

[9] V. Stambolic, A. Suzuki, J. L. De la Pompa et al., "Negative regulation of $\mathrm{PKB} / \mathrm{Akt}$-dependent cell survival by the tumor suppressor PTEN," Cell, vol. 95, no. 1, pp. 29-39, 1998.

[10] H. Sun, R. Lesche, D. M. Li et al., "PTEN modulates cell cycle progression and cell survival by regulating phosphatidylinositol 3,4,5,-trisphosphate and Akt/protein kinase B signaling pathway," Proceedings of the National Academy of Sciences of the United States of America, vol. 96, no. 11, pp. 6199-6204, 1999.

[11] C. Blanco-Aparicio, O. Renner, J. F. M. Leal, and A. Carnero, "PTEN, more than the AKT pathway," Carcinogenesis, vol. 28, no. 7, pp. 1379-1386, 2007.

[12] M. M. Georgescu, K. H. Kirsch, P. Kaloudis, H. Yang, N. P. Pavletich, and H. Hanafusa, "Stabilization and productive positioning roles of the $\mathrm{C} 2$ domain of PTEN tumor suppressor," Cancer Research, vol. 60, no. 24, pp. 7033-7038, 2000.

[13] J. J. Gildea, M. Herlevsen, M. A. Harding et al., "PTEN can inhibit in vitro organotypic and in vivo orthotopic invasion of human bladder cancer cells even in the absence of its lipid phosphatase activity," Oncogene, vol. 23, no. 40, pp. 67886797, 2004.

[14] D. Koul, S. A. Jasser, Y. Lu et al., "Motif analysis of the tumor suppressor gene MMAC/PTEN identifies tyrosines critical for tumor suppression and lipid phosphatase activity," Oncogene, vol. 21, no. 15, pp. 2357-2364, 2002.

[15] D. Maier, G. Jones, X. Li et al., "The PTEN lipid phosphatase domain is not required to inhibit invasion of glioma cells," Cancer Research, vol. 59, no. 21, pp. 5479-5482, 1999.

[16] M. L. Sulis and R. Parsons, "PTEN: from pathology to biology," Trends in Cell Biology, vol. 13, no. 9, pp. 478-483, 2003.

[17] L. P. Weng, J. L. Brown, and C. Eng, "PTEN induces apoptosis and cell cycle arrest through phosphoinositol-3-kinase/Aktdependent and -independent pathways," Human Molecular Genetics, vol. 10, no. 3, pp. 237-242, 2001.

[18] A. Radu, V. Neubauer, T. Akagi, H. Hanafusa, and M. M. Georgescu, "PTEN induces cell cycle arrest by decreasing the level and nuclear localization of cyclin D1," Molecular and Cellular Biology, vol. 23, no. 17, pp. 6139-6149, 2003.

[19] F. B. Furnari, H. J. Su Huang, and W. K. Cavenee, "The phosphoinositol phosphatase activity of PTEN mediates a serum- sensitive G1 growth arrest in glioma cells," Cancer Research, vol. 58, no. 22, pp. 5002-5008, 1998.

[20] M. Tamura, J. Gu, K. Matsumoto, S. I. Aota, R. Parsons, and K. M. Yamada, "Inhibition of cell migration, spreading, and focal adhesions by tumor suppressor PTEN," Science, vol. 280, no. 5369, pp. 1614-1617, 1998.

[21] M. Tamura, J. Gu, E. H. J. Danen, T. Takino, S. Miyamoto, and K. M. Yamada, "PTEN interactions with focal adhesion kinase and suppression of the extracellular matrix-dependent phosphatidylinositol 3-kinase/Akt cell survival pathway," 
Journal of Biological Chemistry, vol. 274, no. 29, pp. 20693 20703, 1999.

[22] M. Tamura, J. Gu, T. Takino, and K. M. Yamada, "Tumor suppressor PTEN inhibition of cell invasion, migration, and growth: differential involvement of focal adhesion kinase and p130(Cas)," Cancer Research, vol. 59, no. 2, pp. 442-449, 1999.

[23] M. Tamura, J. Gu, H. Tran, and K. M. Yamada, "PTEN gene and integrin signaling in cancer," Journal of the National Cancer Institute, vol. 91, no. 21, pp. 1820-1828, 1999.

[24] J. A. Hobert and C. Eng, "PTEN hamartoma tumor syndrome: an overview," Genetics in Medicine, vol. 11, no. 10, pp. 687-694, 2009.

[25] M. C. Hollander, G. M. Blumenthal, and P. A. Dennis, "PTEN loss in the continuum of common cancers, rare syndromes and mouse models," Nature Reviews Cancer, vol. 11, no. 4, pp. 289-301, 2011.

[26] N. M. Monte, K. A. Webster, D. Neuberg, G. R. Dressler, and G. L. Mutter, "Joint loss of PAX2 and PTEN expression in endometrial precancers and cancer," Cancer Research, vol. 70, no. 15, pp. 6225-6232, 2010.

[27] F. Meng, R. Henson, H. Wehbe-Janek, K. Ghoshal, S. T. Jacob, and T. Patel, "MicroRNA-21 regulates expression of the PTEN tumor suppressor gene in human hepatocellular cancer," Gastroenterology, vol. 133, no. 2, pp. 647-658, 2007.

[28] N. Amodio, M. Scrima, L. Palaia et al., "Oncogenic role of the E3 ubiquitin ligase NEDD4-1, a PTEN negative regulator, in non-small-cell lung carcinomas," American Journal of Pathology, vol. 177, no. 5, pp. 2622-2634, 2010.

[29] J. C. Soria, H. Y. Lee, J. I. Lee et al., "Lack of PTEN expression in non-small cell lung cancer could be related to promoter methylation," Clinical Cancer Research, vol. 8, no. 5, pp. 1178-1184, 2002.

[30] C. J. Marsit, S. Zheng, K. Aldape et al., "PTEN expression in non-small-cell lung cancer: evaluating its relation to tumor characteristics, allelic loss, and epigenetic alteration," Human Pathology, vol. 36, no. 7, pp. 768-776, 2005.

[31] Y. E. Whang, X. Wu, H. Suzuki et al., "Inactivation of the tumor suppressor PTEN/MMAC1 in advanced human prostate cancer through loss of expression," Proceedings of the National Academy of Sciences of the United States of America, vol. 95, no. 9, pp. 5246-5250, 1998.

[32] L. Salmena, A. Carracedo, and P. P. Pandolfi, “Tenets of PTEN tumor suppression," Cell, vol. 133, no. 3, pp. 403-414, 2008.

[33] K. Podsypanina, L. H. Ellenson, A. Nemes et al., "Mutation of Pten/Mmacl in mice causes neoplasia in multiple organ systems," Proceedings of the National Academy of Sciences of the United States of America, vol. 96, no. 4, pp. 1563-1568, 1999.

[34] A. Suzuki, J. L. De La Pompa, V. Stambolic et al., "High cancer susceptibility and embryonic lethality associated with mutation of the PTEN tumor suppressor gene in mice," Current Biology, vol. 8, no. 21, pp. 1169-1178, 1998.

[35] L. C. Trotman, M. Niki, Z. A. Dotan et al., "Pten dose dictates cancer progression in the prostate," PLoS Biology, vol. 1, no. 3, article E59, 2003.

[36] A. Di Cristofano, B. Pesce, C. Cordon-Cardo, and P. P. Pandolfi, "Pten is essential for embryonic development and tumour suppression," Nature Genetics, vol. 19, no. 4, pp. 348355, 1998.

[37] A. Alimonti, A. Carracedo, J. G. Clohessy et al., "Subtle variations in Pten dose determine cancer susceptibility," Nature Genetics, vol. 42, no. 5, pp. 454-458, 2010.
[38] K. Stemke-Hale, A. M. Gonzalez-Angulo, A. Lluch et al., "An integrative genomic and proteomic analysis of PIK3CA, PTEN, and AKT mutations in breast cancer," Cancer Research, vol. 68, no. 15, pp. 6084-6091, 2008.

[39] B. T. Hennessy, D. L. Smith, P. T. Ram, Y. Lu, and G. B. Mills, "Exploiting the PI3K/AKT pathway for cancer drug discovery," Nature Reviews Drug Discovery, vol. 4, no. 12, pp. 988-1004, 2005.

[40] J. Brugge, M. C. Hung, and G. B. Mills, "A new mutational aktivation in the PI3K pathway," Cancer Cell, vol. 12, no. 2, pp. 104-107, 2007.

[41] E. K. Yim, G. Peng, H. Dai et al., "Rak functions as a tumor suppressor by regulating PTEN protein stability and function," Cancer Cell, vol. 15, no. 4, pp. 304-314, 2009.

[42] F. Vazquez, S. Ramaswamy, N. Nakamura, and W. R. Sellers, "Phosphorylation of the PTEN tail regulates protein stability and function," Molecular and Cellular Biology, vol. 20, no. 14, pp. 5010-5018, 2000.

[43] Z. M. Sheng, A. Marchetti, F. Buttitta et al., "Multiple regions of chromosome $6 \mathrm{q}$ affected by loss of heterozygosity in primary human breast carcinomas," British Journal of Cancer, vol. 73, no. 2, pp. 144-147, 1996.

[44] T. Mund and H. R. B. Pelham, "Regulation of PTEN/Akt and MAP kinase signaling pathways by the ubiquitin ligase activators Ndfip1 and Ndfip2," Proceedings of the National Academy of Sciences of the United States of America, vol. 107, no. 25, pp. 11429-11434, 2010.

[45] L. C. Trotman, X. Wang, A. Alimonti et al., "Ubiquitination regulates PTEN nuclear import and tumor suppression," Cell, vol. 128, no. 1, pp. 141-156, 2007.

[46] X. Wang, L. C. Trotman, T. Koppie et al., "NEDD4-1 is a proto-oncogenic ubiquitin ligase for PTEN," Cell, vol. 128, no. 1, pp. 129-139, 2007.

[47] F. Fouladkou, T. Landry, H. Kawabe et al., "The ubiquitin ligase Nedd4-1 is dispensable for the regulation of PTEN stability and localization," Proceedings of the National Academy of Sciences of the United States of America, vol. 105, no. 25, pp. 8585-8590, 2008.

[48] X. R. Cao, N. L. Lill, N. Boase et al., "Nedd4 controls animal growth by regulating IGF-1 signaling," Science signaling, vol. 1 , no. 38, article ra5, 2008.

[49] C. Van Themsche, V. Leblanc, S. Parent, and E. Asselin, "Xlinked inhibitor of apoptosis protein (XIAP) regulates PTEN ubiquitination, content, and compartmentalization," Journal of Biological Chemistry, vol. 284, no. 31, pp. 20462-20466, 2009.

[50] Q. L. Deveraux, R. Takahashi, G. S. Salvesen, and J. C. Reed, "X-linked IAP is a direct inhibitor of cell-death proteases," Nature, vol. 388, no. 6639, pp. 300-304, 1997.

[51] A. D. Schimmer, S. Dalili, R. A. Batey, and S. J. Riedl, "Targeting XIAP for the treatment of malignancy," Cell Death and Differentiation, vol. 13, no. 2, pp. 179-188, 2006.

[52] A. Gericke, M. Munson, and A. H. Ross, "Regulation of the PTEN phosphatase," Gene, vol. 374, no. 1-2, pp. 1-9, 2006.

[53] M. Yilmaz and G. Christofori, "Mechanisms of motility in metastasizing cells," Molecular Cancer Research, vol. 8, no. 5, pp. 629-642, 2010.

[54] Y. Zhang, B. Ma, and Q. Fan, "Mechanisms of breast cancer bone metastasis," Cancer Letters, vol. 292, no. 1, pp. 1-7, 2010.

[55] M. Mareel, T. Boterberg, V. Noë et al., "E-cadherin/catenin/ cytoskeleton complex: a regulator of cancer invasion," Journal of Cellular Physiology, vol. 173, no. 2, pp. 271-274, 1997.

[56] X. Wu, K. Hepner, S. Castelino-Prabhu et al., "Evidence for 
regulation of the PTEN tumor suppressor by a membranelocalized multi-PDZ domain containing scaffold protein MAGI-2," Proceedings of the National Academy of Sciences of the United States of America, vol. 97, no. 8, pp. 4233-4238, 2000.

[57] M. C. Subauste, P. Nalbant, E. D. Adamson, and K. M. Hahn, "Vinculin controls PTEN protein level by maintaining the interaction of the adherens junction protein $\beta$-catenin with the scaffolding protein MAGI-2," Journal of Biological Chemistry, vol. 280, no. 7, pp. 5676-5681, 2005.

[58] T. Tolkacheva, M. Boddapati, A. Sanfiz, K. Tsuchida, A. C. Kimmelman, and A. M. L. Chan, "Regulation of PTEN binding to MAGI-2 by two putative phosphorylation sites at threonine 382 and 383," Cancer Research, vol. 61, no. 13, pp. 4985-4989, 2001.

[59] Y. Hu, Z. Li, L. Guo et al., "MAGI-2 Inhibits cell migration and proliferation via PTEN in human hepatocarcinoma cells," Archives of Biochemistry and Biophysics, vol. 467, no. 1, pp. 1-9, 2007.

[60] J. H. Zuo et al., "Activation of EGFR promotes squamous carcinoma SCC10A cell migration and invasion via inducing EMT-like phenotype change and MMP-9-mediated degradation of E-cadherin," Journal of Cellular Biochemistry, vol. 112, no. 9, pp. 2508-2517, 2011.

[61] B. Fingleton, C. C. Lynch, T. Vargo-Gogola, and L. M. Matrisian, "Cleavage of E-cadherin by matrix metalloproteinase-7 promotes cellular proliferation in nontransformed cell lines via activation of RhoA," Journal of Oncology, vol. 2010, Article ID 530745, 11 pages, 2010.

[62] L. Mohamet, M. L. Lea, and C. M. Ward, "Abrogation of Ecadherin-mediated cellular aggregation allows proliferation of pluripotent mouse embryonic stem cells in shake flask bioreactors," PLoS ONE, vol. 5, no. 9, article e12921, 2010.

[63] J. C. M. de Freitas Junior, B. D. R. D. Silva, W. F. de Souza, W. M. de Araújo, E. S. F. W. Abdelhay, and J. A. MorgadoDíaz, "Inhibition of N-linked glycosylation by tunicamycin induces E-cadherin-mediated cell-cell adhesion and inhibits cell proliferation in undifferentiated human colon cancer cells," Cancer Chemotherapy and Pharmacology, vol. 68, no. 1, pp. 227-238, 2011.

[64] S. Rachagani, S. Senapati, S. Chakraborty et al., "Activated Kras G12D is associated with invasion and metastasis of pancreatic cancer cells through inhibition of E-cadherin," British Journal of Cancer, vol. 104, no. 6, pp. 1038-1048, 2011.

[65] R. M. Bremnes, R. Veve, F. R. Hirsch, and W. A. Franklin, "The E-cadherin cell-cell adhesion complex and lung cancer invasion, metastasis, and prognosis," Lung Cancer, vol. 36, no. 2, pp. 115-124, 2002.

[66] M.-T. Lau, C. Klausen, and P. C. K. Leung, "E-cadherin inhibits tumor cell growth by suppressing PI3K/Akt signaling via beta-catenin-Egr1-mediated PTEN expression," Oncogene, vol. 30, no. 24, pp. 2753-2766, 2011.

[67] M. V. Fournier, J. E. Fata, K. J. Martin, P. Yaswen, and M. J. Bissell, "Interaction of E-cadherin and PTEN regulates morphogenesis and growth arrest in human mammary epithelial cells," Cancer Research, vol. 69, no. 10, pp. 45454552, 2009.

[68] L. Kotelevets, J. Van Hengel, E. Bruyneel, M. Mareel, F. Van Roy, and E. Chastre, "The lipid phosphatase activity of PTEN is critical for stabilizing intercellular junctions and reverting invasiveness," Journal of Cell Biology, vol. 155, no. 7, pp. 1129-1135, 2001.
[69] N. B. Adey, L. Huang, P. A. Ormonde et al., "Threonine phosphorylation of the MMAC1/PTEN PDZ binding domain both inhibits and stimulates PDZ binding," Cancer Research, vol. 60, no. 1, pp. 35-37, 2000.

[70] Y. Wu, D. Dowbenko, S. Spencer et al., "Interaction of the tumor suppressor PTEN/MMAC with a PDZ domain of MAGI3, a novel membrane-associated guanylate kinase," Journal of Biological Chemistry, vol. 275, no. 28, pp. 2147721485, 2000.

[71] L. Kotelevets, J. Van Hengel, E. Bruyneel, M. Mareel, F. Van Roy, and E. Chastre, "Implication of the MAGI-1b/PTEN signalosome in stabilization of adherens junctions and suppression of invasiveness," FASEB Journal, vol. 19, no. 1, pp. 115-117, 2005.

[72] X. Peng, L. E. Cuff, C. D. Lawton, and K. A. DeMali, "Vinculin regulates cell-surface E-cadherin expression by binding to $\beta$-catenin," Journal of Cell Science, vol. 123, no. 4, pp. 567-577, 2010.

[73] T. Virolle, E. D. Adamson, V. Baron et al., "The Egr-1 transcription factor directly activates PTEN during irradiationinduced signalling," Nature Cell Biology, vol. 3, no. 12, pp. 1124-1128, 2001.

[74] T. Sano, H. Lin, X. Chen et al., "Differential expression of MMAC/PTEN in glioblastoma multiforme: relationship to localization and prognosis," Cancer Research, vol. 59, no. 8, pp. 1820-1824, 1999.

[75] A. Perren, L. P. Weng, A. H. Boag et al., "Immunohistochemical evidence of loss of PTEN expression in primary ductal adenocarcinomas of the breast," American Journal of Pathology, vol. 155, no. 4, pp. 1253-1260, 1999.

[76] O. Gimm, A. Perren, L. P. Weng et al., "Differential nuclear and cytoplasmic expression of PTEN in normal thyroid tissue, and benign and malignant epithelial thyroid tumors," American Journal of Pathology, vol. 156, no. 5, pp. 1693-1700, 2000.

[77] S. Semba, S. Satake, M. Matsushita, and H. Yokozaki, "Phosphatase activity of nuclear PTEN is required for CDX2mediated intestinal differentiation of gastric carcinoma," Cancer Letters, vol. 274, no. 1, pp. 143-150, 2009.

[78] D. C. Whiteman, X. P. Zhou, M. C. Cummings, S. Pavey, N. K. Hayward, and C. Eng, "Nuclear PTEN expression and clinicopathologic features in a population-based series of primary cutaneous melanoma," International Journal of Cancer, vol. 99, no. 1, pp. 63-67, 2002.

[79] K. S. Jang, Y. S. Song, S. H. Jang et al., "Clinicopathological significance of nuclear PTEN expression in colorectal adenocarcinoma," Histopathology, vol. 56, no. 2, pp. 229-239, 2010.

[80] M. E. Ginn-Pease and C. Eng, "Increased nuclear phosphatase and tensin homologue deleted on chromosome 10 is associated with G0-G1 in MCF-7 cells," Cancer Research, vol. 63, no. 2, pp. 282-286, 2003.

[81] J. H. Chung and C. Eng, "Nuclear-cytoplasmic partitioning of phosphatase and tensin homologue deleted on chromosome 10 (PTEN) differentially regulates the cell cycle and apoptosis," Cancer Research, vol. 65, no. 18, pp. 8096-8100, 2005.

[82] A. I. Jacob, T. Romigh, K. A. Waite, and C. Eng, "Nuclear PTEN levels and G2 progression in melanoma cells," Melanoma research, vol. 19, no. 4, pp. 203-210, 2009.

[83] M. Tachibana, M. Shibakita, S. Ohno et al., "Expression and prognostic significance of PTEN product protein in patients with esophageal squamous cell carcinoma," Cancer, vol. 94, no. 7, pp. 1955-1960, 2002. 
[84] X. P. Zhou, A. Loukola, R. Salovaara et al., "PTEN mutational spectra, expression levels, and subcellular localization in microsatellite stable and unstable colorectal cancers," American Journal of Pathology, vol. 161, no. 2, pp. 439-447, 2002.

[85] S. M. Planchon, K. A. Waite, and C. Eng, "The nuclear affairs of PTEN," Journal of Cell Science, vol. 121, no. 3, pp. 249-253, 2008.

[86] F. Liu, S. Wagner, R. B. Campbell, J. A. Nickerson, C. A. Schiffer, and A. H. Ross, "PTEN enters the nucleus by diffusion," Journal of Cellular Biochemistry, vol. 96, no. 2, pp. 221-234, 2005.

[87] T. Boulikas, "Nuclear localization signals (NLS)," Critical Reviews in Eukaryotic Gene Expression, vol. 3, no. 3, pp. 193227, 1993.

[88] R. Peters, "Fluorescence microphotolysis to measure nucleocytoplasmic transport and intracellular mobility," Biochimica et Biophysica Acta, vol. 864, no. 3-4, pp. 305-359, 1986.

[89] S. Kuersten, M. Ohno, and I. W. Mattaj, "Nucleocytoplasmic transport: ran, beta and beyond," Trends in Cell Biology, vol. 11, no. 12, pp. 497-503, 2001.

[90] A. Gil, A. Andrés-Pons, E. Fernândez et al., "Nuclear localization of PTEN by a Ran-dependent mechanism enhances apoptosis: involvement of an N-terminal nuclear localization domain and multiple nuclear exclusion motifs," Molecular Biology of the Cell, vol. 17, no. 9, pp. 4002-4013, 2006.

[91] M. H. Mossink, A. Van Zon, R. J. Scheper, P. Sonneveld, and E. A. C. Wiemer, "Vaults: a ribonucleoprotein particle involved in drug resistance?" Oncogene, vol. 22, no. 47, pp. 7458-7467, 2003.

[92] Y. Zhenbao, N. Fotouhi-Ardakani, L. Wu et al., "PTEN associates with the vault particles in HeLa cells," Journal of Biological Chemistry, vol. 277, no. 43, pp. 40247-40252, 2002.

[93] J. Torres and R. Pulido, "The tumor suppressor PTEN is phosphorylated by the protein kinase CK2 at its $\mathrm{C}$ terminus. Implications for PTEN stability to proteasome-mediated degradation," Journal of Biological Chemistry, vol. 276, no. 2, pp. 993-998, 2001.

[94] M. M. Georgescu, K. H. Kirsch, T. Akagi, T. Shishido, and H. Hanafusa, "The tumor-suppressor activity of PTEN is regulated by its carboxyl-terminal region," Proceedings of the National Academy of Sciences of the United States of America, vol. 96, no. 18, pp. 10182-10187, 1999.

[95] J. Torres, J. Rodriguez, M. P. Myers et al., "Phosphorylationregulated cleavage of the tumor suppressor PTEN by caspase3. Implications for the control of protein stability and PTENprotein interactions," Journal of Biological Chemistry, vol. 278, no. 33, pp. 30652-30660, 2003.

[96] M. Raftopoulou, S. Etienne-Manneville, A. Self, S. Nicholls, and A. Hall, "Regulation of cell migration by the $\mathrm{C} 2$ domain of the tumor suppressor PTEN," Science, vol. 303, no. 5661, pp. 1179-1181, 2004.

[97] L. Odriozola, G. Singh, T. Hoang, and A. M. Chan, "Regulation of PTEN activity by its carboxyl-terminal autoinhibitory domain," Journal of Biological Chemistry, vol. 282, no. 32, pp. 23306-23315, 2007.

[98] C. J. Chang, D. J. Mulholland, B. Valamehr, S. Mosessian, W. R. Sellers, and H. Wu, "PTEN nuclear localization is regulated by oxidative stress and mediates p53-dependent tumor suppression," Molecular and Cellular Biology, vol. 28, no. 10, pp. 3281-3289, 2008.

[99] J. L. Liu, Z. Mao, T. A. LaFortune et al., "Cell cycle-dependent nuclear export of phosphatase and tensin homologue tumor suppressor is regulated by the phosphoinositide-3-kinase signaling cascade," Cancer Research, vol. 67, no. 22, pp. 11054-11063, 2007.

[100] J.-L. Liu, Z. Mao, G. E. Gallick, and W. K.A. Yung, "AMPK/ TSC2/mTOR-signaling intermediates are not necessary for LKB1-mediated nuclear retention of PTEN tumor suppressor," Neuro-Oncology, vol. 13, no. 2, pp. 184-194, 2011.

[101] M. S. Song, A. Carracedo, L. Salmena et al., "Nuclear PTEN regulates the APC-CDH1 tumor-suppressive complex in a phosphatase-independent manner," Cell, vol. 144, no. 2, pp. 187-199, 2011.

[102] T. Cardozo and M. Pagano, "The SCF ubiquitin ligase: insights into a molecular machine," Nature Reviews Molecular Cell Biology, vol. 5, no. 9, pp. 739-751, 2004.

[103] J. M. Peters, "The anaphase promoting complex/cyclosome: a machine designed to destroy," Nature Reviews Molecular Cell Biology, vol. 7, no. 9, pp. 644-656, 2006.

[104] J. Pines, "Mitosis: a matter of getting rid of the right protein at the right time," Trends in Cell Biology, vol. 16, no. 1, pp. 55-63, 2006.

[105] M. Sullivan and D. O. Morgan, "Finishing mitosis, one step at a time," Nature Reviews Molecular Cell Biology, vol. 8, no. 11, pp. 894-903, 2007.

[106] S. M. Hammond, "RNAi, microRNAs, and human disease," Cancer Chemotherapy and Pharmacology, vol. 58, pp. s63s68, 2006.

[107] V. Ambros, "MicroRNA pathways in flies and worms: growth, death, fat, stress, and timing," Cell, vol. 113, no. 6, pp. 673676, 2003.

[108] S. Bagga, "Posttransplant lymphoproliferative disease in a pediatric patient as seen on PET/CT scan," Clinical Nuclear Medicine, vol. 32, no. 7, pp. 553-554, 2007.

[109] D. P. Bartel, "MicroRNAs: genomics, biogenesis, mechanism, and function," Cell, vol. 116, no. 2, pp. 281-297, 2004.

[110] K. K. H. Farh, A. Grimson, C. Jan et al., "The widespread impact of mammalian microRNAs on mRNA repression and evolution," Science, vol. 310, no. 5755, pp. 1817-1821, 2005.

[111] D. S. Schwarz, G. Hutvágner, T. Du, Z. Xu, N. Aronin, and P. D. Zamore, "Asymmetry in the assembly of the RNAi enzyme complex," Cell, vol. 115, no. 2, pp. 199-208, 2003.

[112] T. Li, D. Li, J. Sha, P. Sun, and Y. Huang, "MicroRNA-21 directly targets MARCKS and promotes apoptosis resistance and invasion in prostate cancer cells," Biochemical and Biophysical Research Communications, vol. 383, no. 3, pp. 280-285, 2009.

[113] Z. Liang, H. Wu, S. Reddy et al., "Blockade of invasion and metastasis of breast cancer cells via targeting CXCR4 with an artificial microRNA," Biochemical and Biophysical Research Communications, vol. 363, no. 3, pp. 542-546, 2007.

[114] L. X. Yan, X. F. Huang, Q. Shao et al., "MicroRNA miR21 overexpression in human breast cancer is associated with advanced clinical stage, lymph node metastasis and patient poor prognosis," RNA, vol. 14, no. 11, pp. 2348-2360, 2008.

[115] S. F. Tavazoie, C. Alarcón, T. Oskarsson et al., "Endogenous human microRNAs that suppress breast cancer metastasis," Nature, vol. 451, no. 7175, pp. 147-152, 2008.

[116] S. Valastyan, F. Reinhardt, N. Benaich et al., "A pleiotropically acting microRNA, miR-31, inhibits breast cancer metastasis," Cell, vol. 137, no. 6, pp. 1032-1046, 2009.

[117] X. F. Li, P. J. Yan, and Z. M. Shao, "Downregulation of miR$193 \mathrm{~b}$ contributes to enhance urokinase-type plasminogen activator ( $\mathrm{uPA}$ ) expression and tumor progression and invasion in human breast cancer," Oncogene, vol. 28, no. 44, pp. 3937-3948, 2009. 
[118] J. G. Zhang, J. J. Wang, F. Zhao, Q. Liu, K. Jiang, and G. H. Yang, "MicroRNA-21 (miR-21) represses tumor suppressor PTEN and promotes growth and invasion in non-small cell lung cancer (NSCLC)," Clinica Chimica Acta, vol. 411, no. 1112, pp. 846-852, 2011.

[119] X. Ma, M. Kumar, S. N. Choudhury et al., "Loss of the miR-21 allele elevates the expression of its target genes and reduces tumorigenesis," Proceedings of the National Academy of Sciences of the United States of America, vol. 108, no. 25, pp. 10144-10149, 2011.

[120] L. Qi, J. Bart, L. P. Tan et al., "Expression of miR-21 and its targets (PTEN, PDCD4, TM1) in flat epithelial atypia of the breast in relation to ductal carcinoma in situ and invasive carcinoma," BMC Cancer, vol. 9, article 163, 2009.

[121] M. Folini, P. Gandellini, N. Longoni et al., "miR-21: an oncomir on strike in prostate cancer," Molecular Cancer, vol. 9, article 12, 2010.

[122] H. Yang, W. Kong, L. He et al., "MicroRNA expression profiling in human ovarian cancer: miR-214 induces cell survival and cisplatin resistance by targeting PTEN," Cancer Research, vol. 68, no. 2, pp. 425-433, 2008.

[123] A. M. Cheng, M. W. Byrom, J. Shelton, and L. P. Ford, "Antisense inhibition of human miRNAs and indications for an involvement of miRNA in cell growth and apoptosis," Nucleic Acids Research, vol. 33, no. 4, pp. 1290-1297, 2005.

[124] X. Xiong, H.-Z. Ren, M.-H. Li, J.-H. Mei, J.-F. Wen, and C.L. Zheng, "Down-regulated miRNA-214 induces a cell cycle G1 arrest in gastric cancer cells by up-regulating the PTEN protein," Pathology and Oncology Research, vol. 17, no. 4, pp. 931-937, 2011.

[125] C. Wang, Z. Bian, D. Wei, and J.-G. Zhang, "miR-29b regulates migration of human breast cancer cells," Molecular and Cellular Biochemistry, vol. 352, no. 1-2, pp. 197-207, 2011.

[126] C. Zhang, C. Kang, P. Wang et al., "MICRORNA-221 and -222 regulate radiation sensitivity by targeting the PTEN pathway," International Journal of Radiation Oncology Biology Physics, vol. 80, no. 1, pp. 240-248, 2011. 

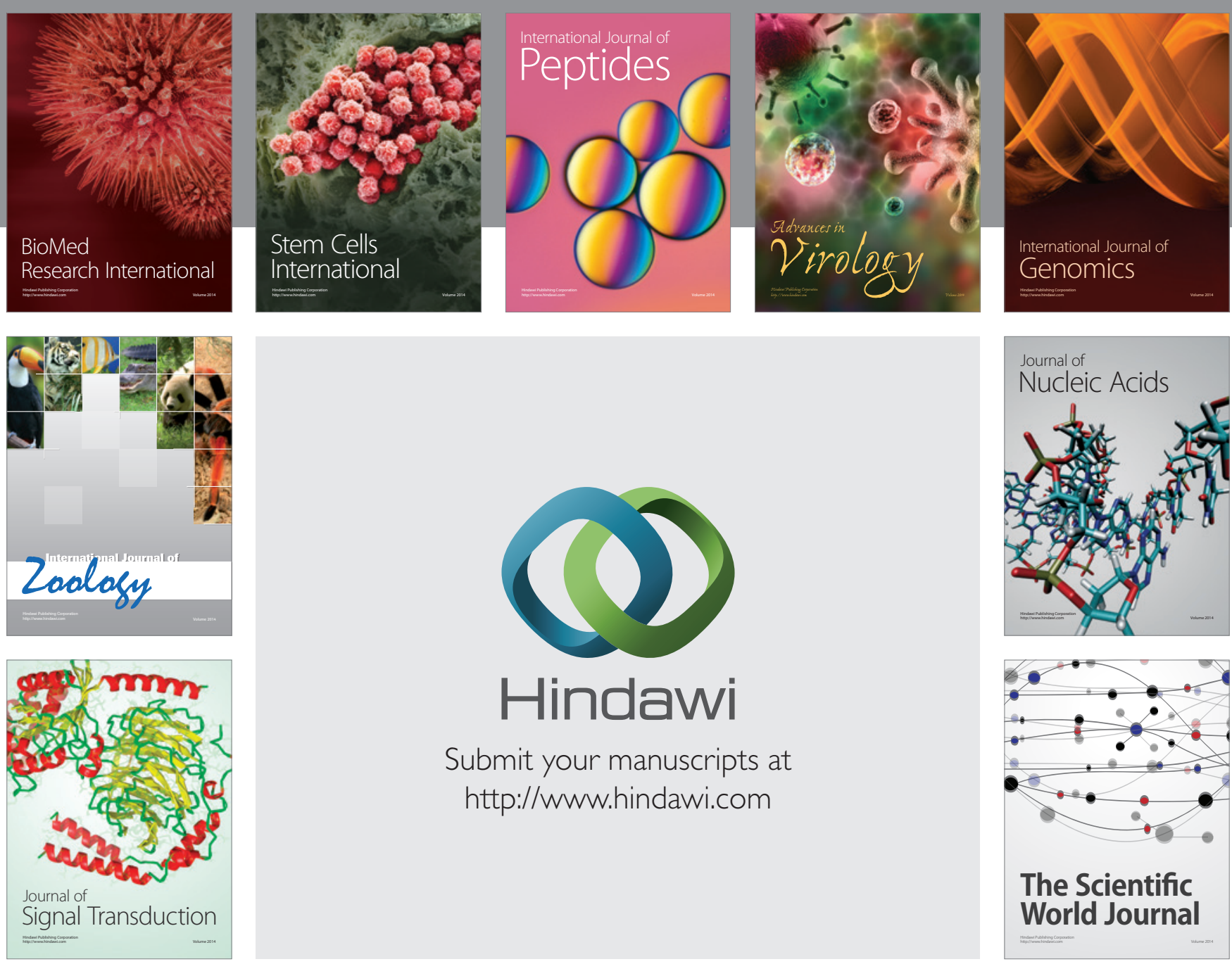

Submit your manuscripts at

http://www.hindawi.com
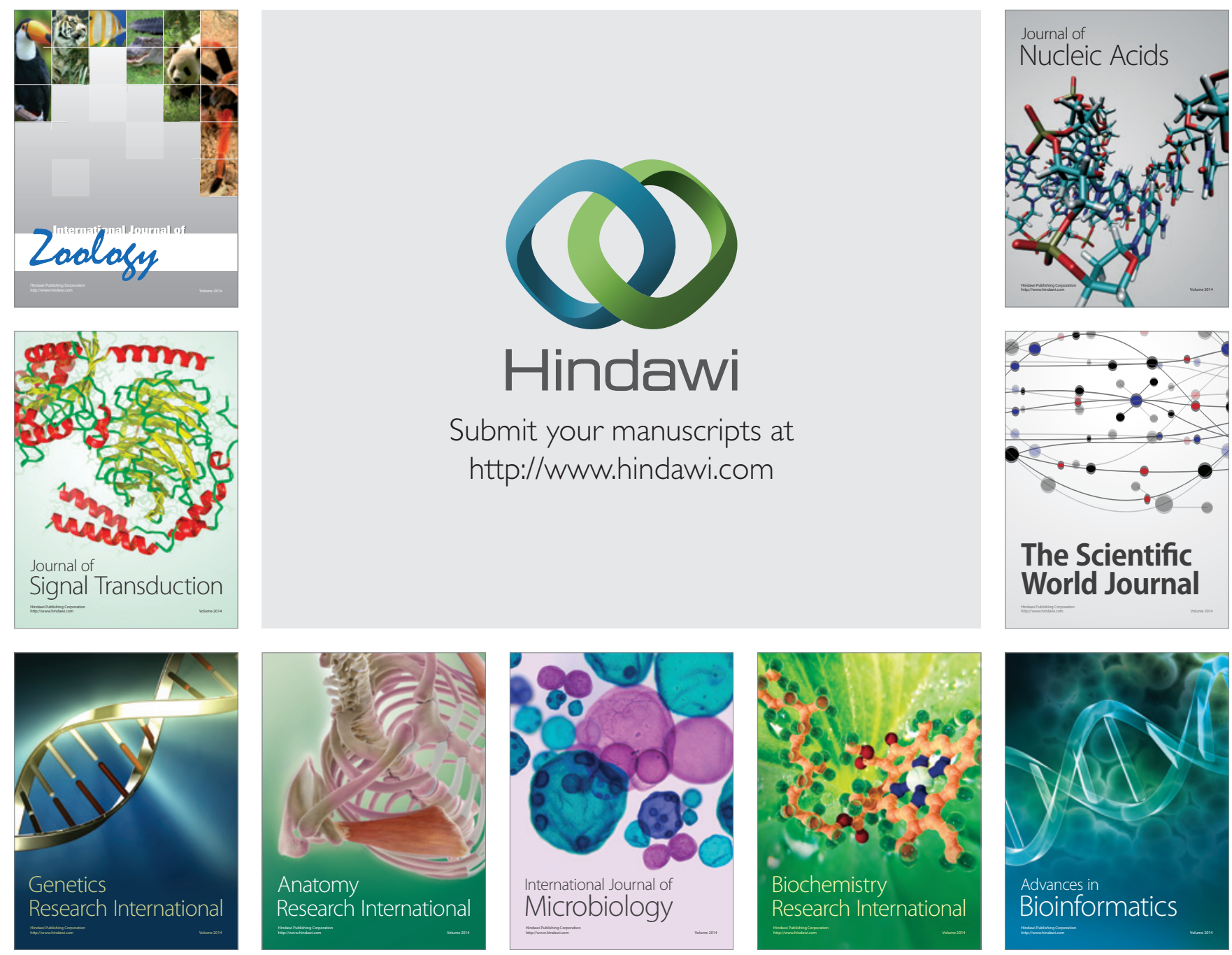

The Scientific World Journal
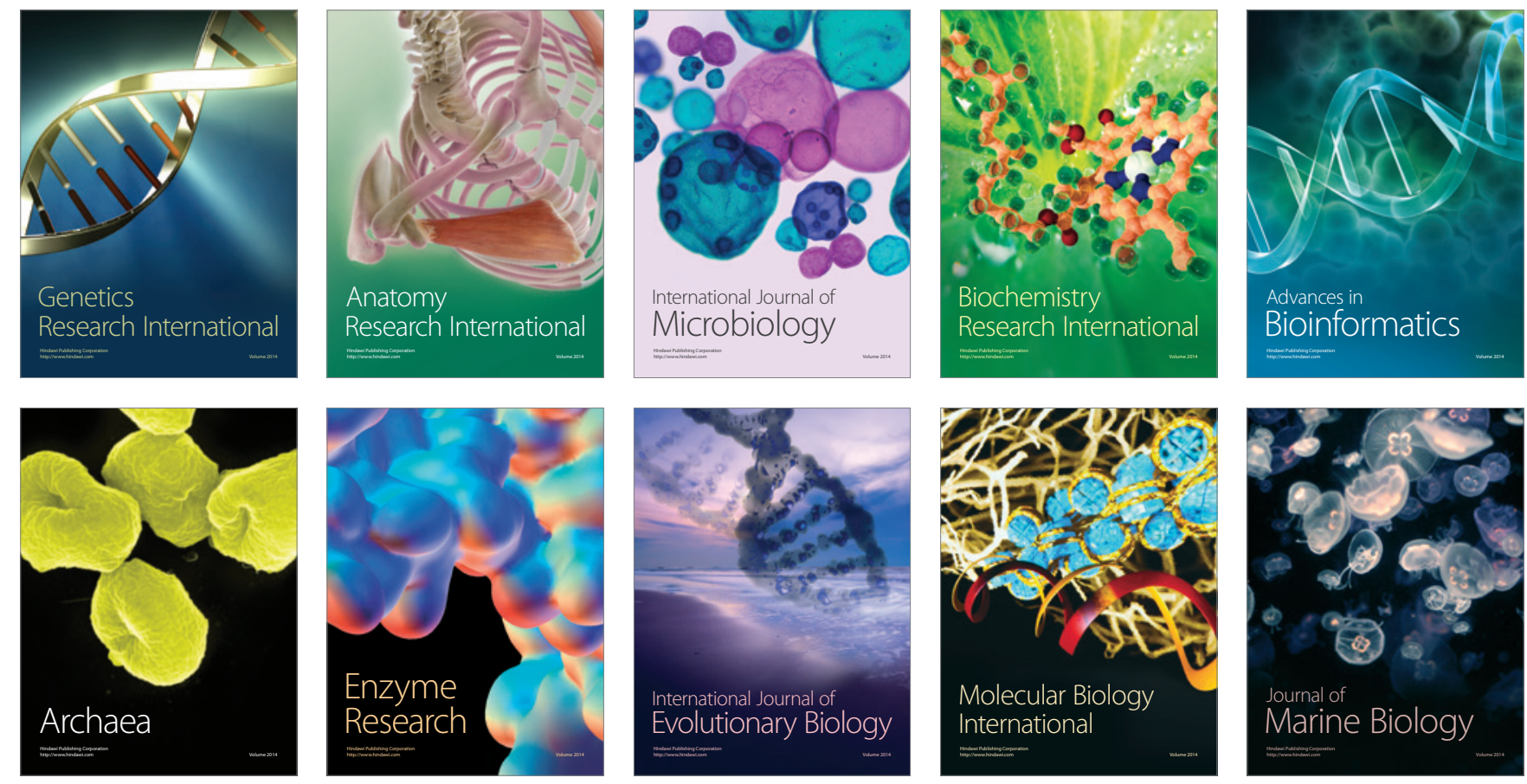